\title{
Aging Wire Insulation Assessment by Phase Spectrum Examination of Ultrasonic Guided Waves
}

\author{
Robert F. Anastasi ${ }^{1}$ and Eric I. Madaras ${ }^{2}$ \\ ${ }^{1}$ U.S. Army Research Laboratory, Vehicle Technology Directorate, AMSRL-VT-S, Nondestructive \\ Evaluation Sciences Branch, NASA Langley Research Center, \\ Hampton, VA 23681 \\ ${ }^{2}$ NASA Langley Research Center, Nondestructive Evaluation Sciences Branch, \\ Hampton, VA 23681
}

\begin{abstract}
Wire integrity has become an area of concern to the aerospace community including DoD, NASA, FAA, and Industry. Over time and changing environmental conditions, wire insulation can become brittle and crack. The cracks expose the wire conductor and can be a source of equipment failure, short circuits, smoke, and fire. The technique of using the ultrasonic phase spectrum to extract material properties of the insulation is being examined. Ultrasonic guided waves will propagate in both the wire conductor and insulation. Assuming the condition of the conductor remains constant then the stiffness of the insulator can be determined by measuring the ultrasonic guided wave velocity. In the phase spectrum method the guided wave velocity is obtained by transforming the time base waveform to the frequency domain and taking the phase difference between two waveforms. The result can then be correlated with a database, derived by numerical model calculations, to extract material properties of the wire insulator. Initial laboratory tests were performed on a simple model consisting of a solid cylinder and then a solid cylinder with a polymer coating. For each sample the flexural mode waveform was identified. That waveform was then transformed to the frequency domain and a phase spectrum was calculated from a pair of waveforms. Experimental results on the simple model compared well to numerical calculations. Further tests were conducted on aircraft or mil-spec wire samples, to see if changes in wire insulation stiffness can be extracted using the phase spectrum technique.
\end{abstract}

Keywords: wire insulation, ultrasonic, guided waves, phase spectrum

\section{INTRODUCTION}

Electrical wiring in most modern day equipment is subjected to heat, cold, moisture, and vibrations, which can eventually cause the wire insulation and even the wire conductor to fail. In most cases these environmental and operational conditions are modest, but in some cases these conditions are extreme and can cause the insulation to become brittle and crack. The cracks expose the underlying wire conductor and become a potential source for short circuits and fire. In critical situations wire inspections are done visually, but often after-the-fact, in response to an instrument or system failure. Visual inspection may find the cracks and burns, but this method offers little quantitative information about the condition of the wire insulation prior to failure.

Ultrasound can be used to obtain the desired quantitative information about the wire insulation. For this purpose, insulated wire may be considered a cylindrical wave-guide or more descriptively a clad rod, where the wire conductor is the core and the wire insulation is the cladding. A number of authors have examined acoustic wave propagation in a cylindrical geometry [1-5] and for detailed analysis the reader is referred to these papers. Some applications of ultrasonic guided waves include material testing or characterization of wire or fibers $[6,7]$, and for use as ultrasonic

SPIE's $8^{\text {th }}$ International Symposium on NDE for Health Monitoring and Diagnostics, Conference 5046, Paper 29, San Diego, CA, 2-6 March 2003 
delay lines. In general many acoustic wave modes will propagate in a cylindrical shape and in part, be a function of material properties, geometry, frequency, propagation order, and circumferential order. Two modes in the low frequency regime are the axisymmetric and flexural modes. The axisymmetric mode is the first signal that arrives in an ultrasonic transmit/receiver arrangement and is nearly non-dispersive in the low-frequency regime. A traditional timeof-flight measurement was previously used in examining the propagation of this mode in heat damaged wire insulation, $[8,9]$. Results showed a correlation between the axisymmetric mode phase velocity and heat-damaged condition of the wire insulation. A drawback in this time-of-flight measurement is that it is tedious and extremely times consuming because a constant phase point from signal-to-signal is followed. Also, the amplitude of the axisymmetric mode signal is smaller in magnitude than the flexural mode signal, due in part to the loading geometry. Thus it requires increasing the electronic signal gain. Taking advantage of the larger amplitude signal should improve the signal analysis, but a simple time of flight measurement can not be used to evaluate the flexural mode phase velocity because this mode is dispersive in nature. A Fourier phase method of extracting phase velocities from this type of dispersive signal was described and demonstrated by Sachse and Pao, [10], and used by a number of other authors. [11-14]. These authors applied the Fourier phase method to Lamb wave signals in metallic and laminated composite materials. In this paper the Fourier phase method is investigated for application on small diameter cylindrical geometry and in particular, electrical wire. Flexural mode dispersion curves are obtained for a wire model and for a mil-spec baseline and heat damaged samples.

\section{EXPERIMENTS}

The experimental system is schematically shown in Figure 1. This system consists of two piezoelectric transducers, ultrasonic pulse generator, ultrasonic pre-amp, and oscilloscope. The transducers were low frequency, broadband acoustic emission transducers with a bandwidth of $50 \mathrm{kHz}$ to $1.5 \mathrm{MHz}$. The signal from the ultrasonic receiver is first fed through a pre-amplifier with a $20 \mathrm{kHz}$ to $2 \mathrm{MHz}$ bandwidth and a 40 or $60 \mathrm{~dB}$ gain and then through another amplifier with a maximum gain of $42 \mathrm{~dB}$ and a bandwidth set at $10 \mathrm{kHz}$ to $300 \mathrm{kHz}$. The output of the amplifier was recorded by an 8-bit/500 MHz digitizing oscilloscope. The signal was averaged 100 times to improve signal to noise and then recorded for later analysis. The transducers were mechanically attached to the rod or wire as shown in Figure 2. The clamp face opposite the transducer had a groove machined in it, to hold the wire along the center of the transducer surface. During measurements, a wire was held on a $30-\mathrm{cm}$ long optical rail while the transducers were clipped on the wire at various separations. The wires were nominally $60 \mathrm{~cm}$ long and the ends of the wires were clamped to hold the samples while measurements were taken.

In the Fourier phase method an elastic wave is generated and detected at two different distances along the rod or wire. For each signal the flexural mode wave is windowed and then the phase $(\varphi)$ of this windowed portion of the signal is obtained by performing a Fourier transform. The phases were unwrapped to remove any $2 \pi n$ uncertainties and then the phase difference $(\Delta \varphi)$ is obtained. The phase velocity $\left(v_{\mathrm{ph}}\right)$ as a function of frequency (f) can be found to be [10]

$$
v_{p h}(f)=\frac{2 \pi f d}{\Delta \phi}
$$

where $\mathrm{d}$ is the distance between the two receiver locations.

To validate the method, a flexural mode dispersion curve for a brass rod was obtained experimentally using the Fourier method and calculated numerically. The calculations were performed using a commercially available software package that calculates dispersion curves in plate and cylindrical geometry. The brass rod was then covered with a heat shrink tubing to simulate the insulation on an electrical wire and measurements and calculations were repeated. In the modeling calculations it is assumed there was perfect coupling between the brass rod and heat shrink tubing. Material properties and geometry of the brass rod and heat shrink tubing used in the calculations are shown in Table I. The longitudinal velocity of the heat shrink tubing was measured using ultrasonic pulse echo methods and the density was measured through volume and mass measurements of a small sample. Other values were estimated using these measurements and tabulated text book values for thermoplastic material [15]. 
TABLE I. Brass and Heat Shrink Tubing Material Properties and Radius.

\begin{tabular}{|l|l|l|}
\hline Material & Brass & Heat Shrink Tubing \\
\hline Young's Modulus, $(\mathrm{GPa})$ & 108.94 & 0.72 \\
\hline Density, $\left(\mathrm{kg} / \mathrm{m}^{3}\right)$ & 8500 & 958 \\
\hline Poission's Ratio & 0.35 & 0.46 \\
\hline Long. Velocity, $(\mathrm{m} / \mathrm{s})$ & 4535 & 1868 \\
\hline Shear Velocity, $(\mathrm{m} / \mathrm{s})$ & 2178 & 510 \\
\hline Rayleigh Velocity, $(\mathrm{m} / \mathrm{s})$ & 2036 & 484 \\
\hline Radius, $(\mathrm{mm})$ & 1.59 & 2.175 \\
\hline
\end{tabular}

Next, measurements were performed on electrical wire samples. The samples were mil-spec MIL-W-22759/34 16gauge Teflon ${ }^{\circledR}$ coated wire. The wire conductor was composed of 19 strands of tin-coated copper. Dimensions and other characteristic of the electrical wire are given in Table II. This electrical wire was examined in a baseline condition (undamaged) and in a heat-damaged or aged condition. Heat damaging or oven aging was performed by heating the wire in an oven for 100 hour at $270{ }^{\circ} \mathrm{C}$. In its baseline condition the electrical wire insulation was white and flexible. After oven aging the insulation was gray and it remained flexible, but felt stiffer compare to the baseline. For numerical calculations the electrical wire was modeled with a core of solid copper and a cladding of Teflon ${ }^{\circledR}$. Properties and radius used in the calculations are shown in Table III. The properties are typical text book values.

TABLE II. Specifications of 16-gauge MIL-W-22759/34 wire

\begin{tabular}{|l|l|}
\hline Overall Diameter, $(\mathrm{mm})$ & 1.90 \\
\hline Wire Bundle Dia., $(\mathrm{mm})$ & 1.33 \\
\hline Insulation Thickness, $(\mathrm{mm})$ & 0.28 \\
\hline Wire Strands per Bundle & 19 \\
\hline Wire Strand Gauge & 29 \\
\hline
\end{tabular}

TABLE III. Material Properties and Radius used in numerical model of electrical wire.

\begin{tabular}{|l|l|l|}
\hline Material & Copper & Teflon \\
\hline Young's Modulus, $(\mathrm{GPa})$ & 122.70 & 1.864 \\
\hline Density, $\left(\mathrm{kg} / \mathrm{m}^{3}\right)$ & 8900 & 2200 \\
\hline Poission's Ratio & 0.35 & 0.40 \\
\hline Long. Velocity, $(\mathrm{m} / \mathrm{s})$ & 4700 & 1350 \\
\hline Shear Velocity, $(\mathrm{m} / \mathrm{s})$ & 2260 & 550 \\
\hline Radius, $(\mathrm{mm})$ & 0.66 & 0.95 \\
\hline
\end{tabular}

\section{RESULTS AND DISCUSSION}

Theoretical dispersion curves for the axisymmetric and flexural low-order modes are shown in Figure 3. These dispersion curves are for an uncoated and coated brass rod of which the material properties and geometry values used to calculate these curves were given in Table I. This figure, illustrate the significant effect of the coating. This effect decreases the range of frequencies where dispersion is most noticeable and decreases the magnitude of the phase velocity. This effect was shown to be a function of the coating thickness [16]. The high-frequency phase velocity limit is the Rayleigh surface wave velocity and for the uncoated and coated rod this is $2100 \mathrm{~m} / \mathrm{s}$ and $510 \mathrm{~m} / \mathrm{s}$ respectively. Experimental measurements were taken on the uncoated and coated brass rod and the flexural mode dispersion curves were obtained as described above. The results shown in Figure 4 are compared with the calculated flexural mode 
dispersion curves that were shown in Figure 3. Overall, the experimental measurements follow the trend of the theoretical curves and illustrate the effect of the coating and validate the Fourier method procedures. The difference between the theoretical and experimental curves may be due to differences between actual and textbook material property values and between actual boundary conditions and the assumed perfect coupling of the core and cladding.

Experimental and theoretical flexural mode dispersion curves for the baseline and oven aged electrical wire are shown in Figure 5. Values in Table III were used to calculate the theoretical baseline dispersion curve. The experimental data follows the trend of this theoretical curve. The theoretical oven aged dispersion curve was calculated using a Young's Modulus of 4.0GPa, while density, and Poissons's Ratio remained constant and longitudinal and shear wave velocity were recalculated according to elastic relationships. The value of $4.0 \mathrm{GPa}$ was chosen to make the theoretical curve follow the experimental data and illustrate stiffening of the wire insulation. Although these curves don't follow the theoretical calculations exactly, in part due to the reasons given above, plus the model used a solid core while the wire core consisted of a bundle of small diameter wires, they do show a difference that may be associated with material stiffness.

Although the measurements were easy to obtain, isolating the flexural mode proved more difficult because of the addition of higher wave modes. The fact that boundary conditions may have been less then perfect may have also compounded the difficulty in interpreting signals. The frequency bandwidth of the signals seemed limited compared to the bandwidth of the transducers and this seemed to limit the experimental results. The small frequency bandwidth may in part be due to the coating. The coating in general is a plastic material that attenuates the propagation of ultrasound. In this case the lower frequencies propagate because they have more energy than the higher frequencies. The composition of the core, a bundle of small diameter wires, may have also attributed to signal attenuation by scattering and dephasing of the ultrasonic signal. To overcome these difficulties and improve wire insulation characterization it may be possible to generate only surface waves instead of relying on the natural mode conversion of the transducer generated longitudinal waves. Since the dispersion curves showed that in the high-frequency regime Rayleigh surface waves propagate, it may be beneficial to use this wave mode for measuring wire insulation properties.

\section{REFERENCES}

1. T.R. Meeker, and A.H. Meitzler., "Guided Wave Propagation in Elongated Cylinders and Plates," in Physical Acoustics Principles and Methods, edited by W.P. Mason, Academic Press, NY, Vol. 1, Part A, 1964.

2. R.N. Thurston, J. Acoust. Soc. Am., 64, 1, 1-37, (1978).

3. H.D. McNiven, J.L. Sackman, and A.H. Shah, J. Acoust. Soc. Am., 35, 10, 1602-1609,(1963).

4. H.N. Abramson, J. Acoust. Soc. Am., 29, 1, 42-46, (1957).

5. J.L. Rose, "Ultrasonic Waves in Solid Media," Cambridge University Press, NY, 1999.

6. E.I. Madaras, T. Kohl, and W.P. Rogers, "Material Property Characterization and Pulse Propagation in Thin Drawn Wire Waveguides," IEEE Ultrasonics Symposium-1992, pp. 957-962.

7. E.I. Madaras, T. Kohl, W.P. Rogers, J. Acoust. Soc. Am., 97, 1, 252-261, (1995).

8. R.F. Anastasi and E.I. Madaras, "Ultrasonic Guided Waves For Aging Wire Insulation," The $28^{\text {th }}$ Annual Review of Progress in QNDE, Eds. D.O. Thompson and D.E. Chimenti, American Institute of Physics, Vol. 21, pp 255-261, 2002.

9. E.I. Madaras and R.F. Anastasi, "Comparison of Ultrasound with Tensile Testing of Thermally Damaged Polyimide Insulated Wire (Mil-W-81381)," The $29^{\text {th }}$ Annual Review of Progress in Quantitative NDE, Western Washington University, Bellingham, Washington, July 14-19, 2002.

10. W. Sachse, W. and Y. Pao, "On the determination of Phase and Group Velocities of Dispersive Waves in Solids," J. Appl. Phys, 49(8), August 1978.

11. N.A. Schumacher, C.P. Burger, and P.H. Gien, "A Laser-Based Investigation of Higher-Order Modes in Transient Lamb Waves,” J. Acoust. Soc. Am., 93(5), pp 2981-2984, May 1993.

12. W.H. Prosser and M.R. Gorman, "Plate Mode Velocities in Graphite/Epoxy Plates," J. Acoust. Soc. Am. 96, pp 902-907, August 1994.

13. M. Veidt and M. Sayir, "Experimental Evaluation of Global Composite Laminate Stiffness by Structural Wave Propagation," Journal of Composite Materials, Vol. 24, pp 688-706, July 1990.

14. D. Alleyne and P. Cawley, "A Two-Dimensional Fourier Transform Method for the Measurement of Propagating Multimode Signals," J. Acoust. Soc. Am. 89(3), pp 1159-1168, March 1991.

15. S. V. Gangal and W. Grot, Tetrafluoroetheylene Polymers, Encyclopedia Of PolymerScience and Engineering, 16, 577-648, 1989.

16. R.C. Reuter, Jr., "Dispersion of Flexural Waves in Circular Bimaterial Cylinders- Theoretical Treatment," J. Acoust. Soc. Am. Vol. 46, No. 3, pp 643-648, 1968. 


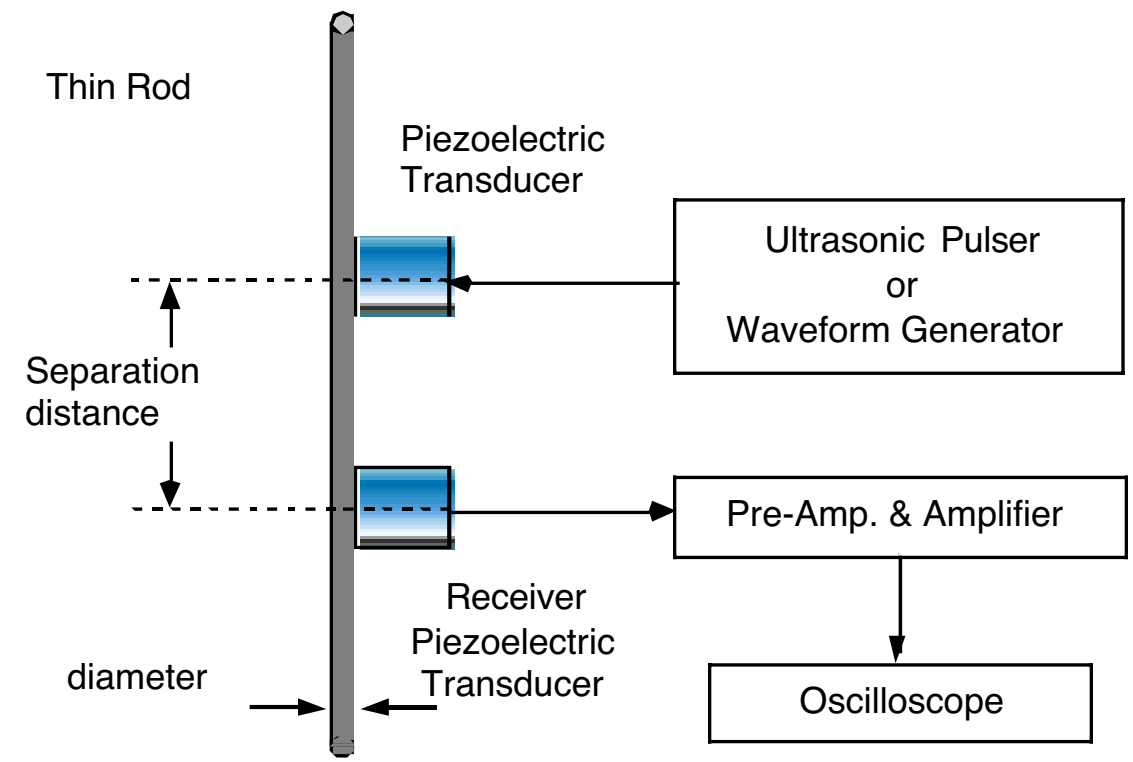

Fig. 1: Schematic of experimental setup.

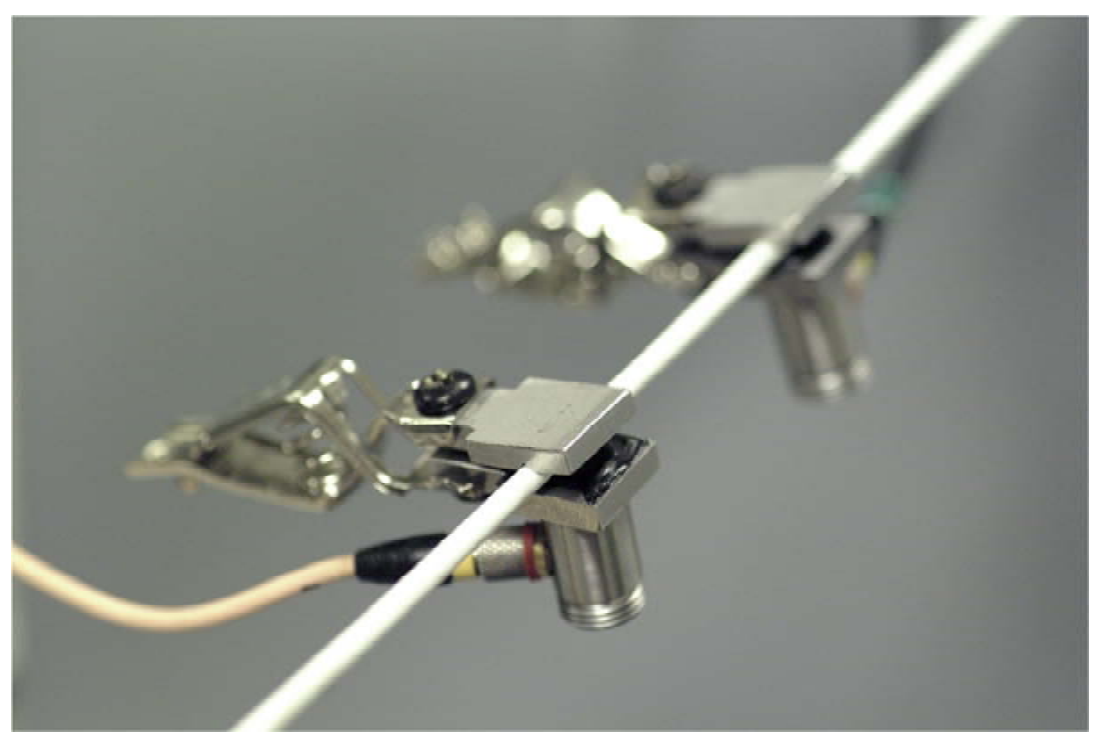

Fig. 2: Ultrasonic Transducers Clipped to Insulated Wire. 


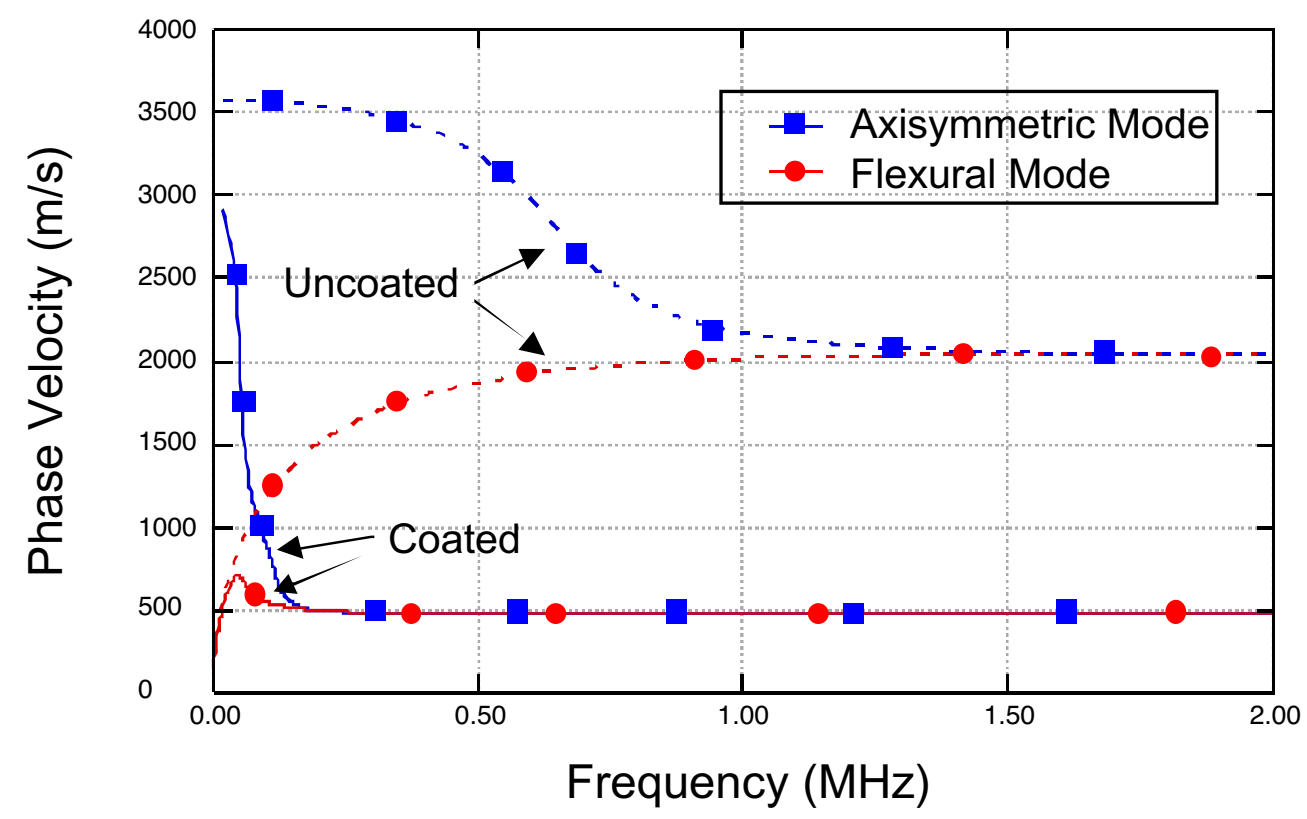

Fig. 3: Theoretical axisymmetric and flexural dispersion curves for uncoated and plastic coated brass rod.

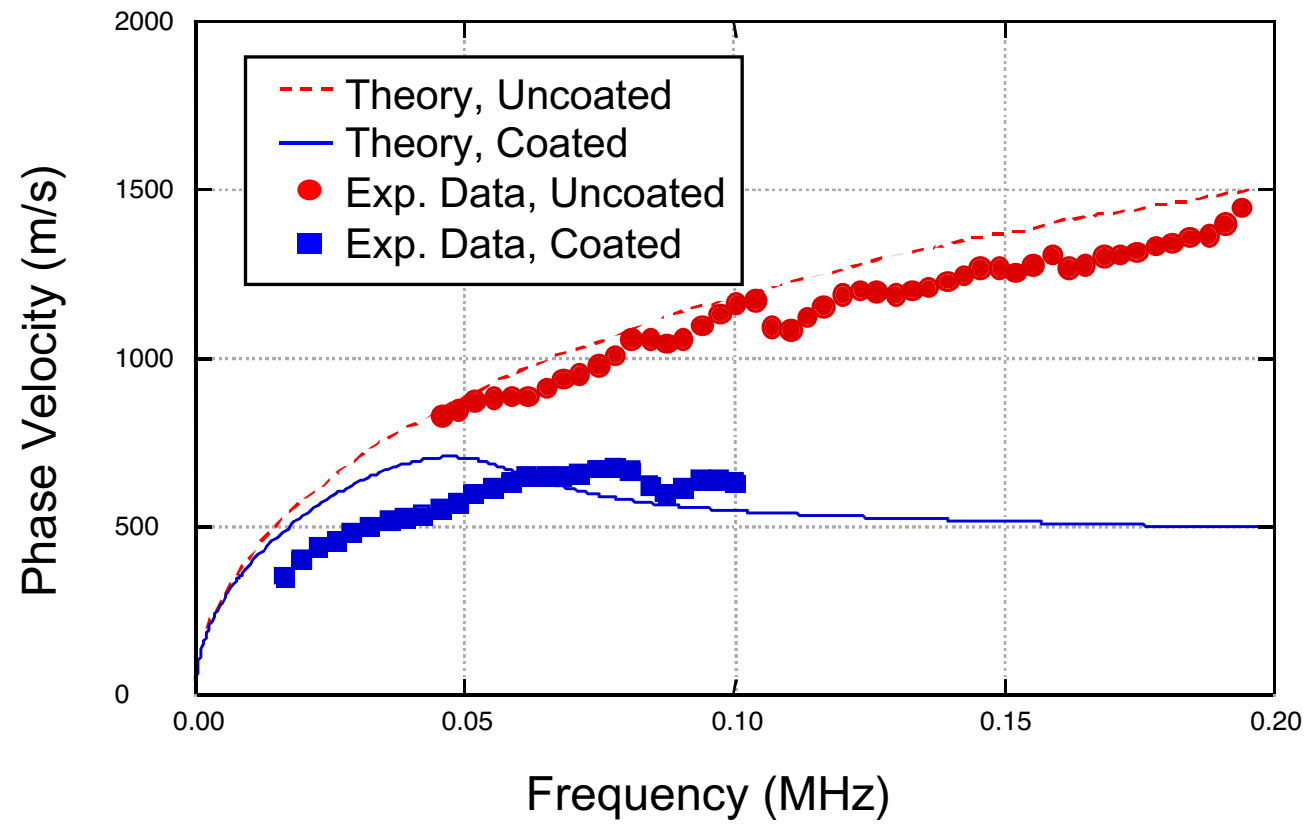

Fig. 4: Comparison of theoretical and experimental flexural mode dispersion curves for uncoated and plastic coated brass rod. 


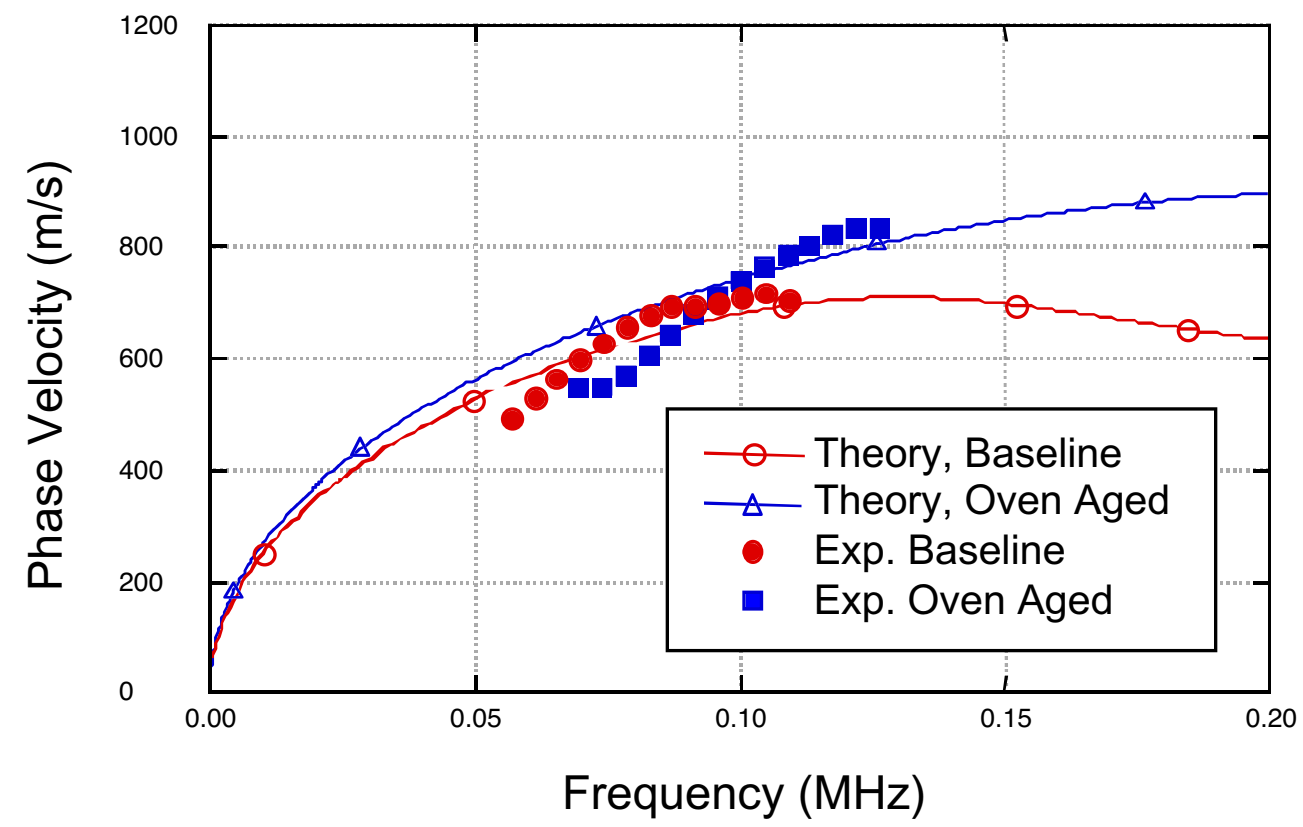

Fig. 5: Theoretical and experimental flexural mode dispersion curves for baseline and oven aged electrical wire. 